\title{
Pattern recognition of surface electromyography signal based on wavelet coefficient entropy
}

\author{
Xiao Hu, Ying Gao, Wai-Xi Liu \\ School of Mechanical and Electric Engineering, Guangzhou University, Guangzhou Higher Education Mega Center, Guangzhou, \\ China; huxiaocz@163.com
}

Received 26 April 2009; revised 15 May 2009; accepted 21 May 2009.

\begin{abstract}
This paper introduced a novel, simple and effective method to extract the general feature of two surface EMG (electromyography) signal patterns: forearm supination (FS) surface EMG signal and forearm pronation (FP) surface EMG signal. After surface EMG (SEMG) signal was decomposed to the fourth resolution level with wavelet packet transform (WPT), its whole scaling space (with frequencies in the interval $(0 \mathrm{~Hz}, 500 \mathrm{~Hz}])$ was divided into16 frequency bands (FB). Then wavelet coefficient entropy (WCE) of every FB was calculated and correspondingly marked with WCE(n) (from the nth FB, $n=1,2, \ldots 16)$. Lastly, some WCE( $n)$ were chosen to form WCE feature vector, which was used to distinguish FS surface EMG signals from FP surface EMG signals. The result showed that the WCE feather vector consisted of WCE(7) $(187.25 \mathrm{~Hz}, 218.75 \mathrm{~Hz})$ and WCE(8) $(218.75 \mathrm{~Hz}, 250 \mathrm{~Hz})$ can more effectively recognize FS and FP patterns than other WCE feature vector or the WPT feature vector which was gained by the combination of WPT and principal components analysis.
\end{abstract}

Keywords: Surface EMG Signal; Wavelet Packet Transform; Entropy; Pattern Recognition

\section{INTRODUCTION}

Due to its noninvasive measurement, surface EMG (SEMG) signal has been widely applied in many fields [1-3]. In this paper, the SEMG signal recorded from the skin surface over limb muscles in the process of the limb actions is called action SEMG (ASEMG) signal. Containing the electrical and functional properties of limb muscle contraction [4] and providing the information about the neuromuscular activity from which ASEMG signal originates [5], ASEMG signal has been widely researched and used in rehabilitation and the controls of prosthetic devices for individuals with amputations or congenitally deficient limbs [6].

ASEMG signal is constituted by many motor unit action potentials (MUAPs) from many recruited motor units under surface electrode and noise [7]. There are three layers of tissues between motor unit and surface electrode: muscle layer, fat layer and skin layer [8]. The tissues introduce low-pass filter effect on MUAPs [9]. MUAPs from the motor units closer to surface electrode distribute in higher frequency band, and MUAPs from the motor units farther (deeper) fall in lower frequency band. The contributions of different muscles to the spectral energy distribution of ASEMG signal are different, because some muscles influence on the high-frequency spectrum and other muscles influence on the low-frequency spectrum. Therefore, the spectral analysis may be an effective means for disclosing the electrical and functional properties of muscle contraction and obtaining the diagnostic information about muscles.

So far, many methods such as time-frequency distribution [5] have been used to analyze the spectral energy distribution of ASEMG signal. However, due to its noninvasive measurement, there was still an obvious motivation to explore some more effective algorithms to extract the features from shorter surface EMG signal and to reduce the error identification rate.

This paper introduced a novel and simple algorithm based on wavelet transform. Firstly, SEMG signal was decomposed to the fourth resolution level with WPT, its whole scaling space (with frequencies in the interval $(0 \mathrm{~Hz}, 500 \mathrm{~Hz}])$ was divided intol6 frequency bands (FB). Secondly, WCE of every FB was calculated and correspondingly marked with WCE(n) (from the $n$th FB, $\mathrm{n}=1,2, \ldots 16)$. Lastly, some WCE(n) were chosen to form WCE feature vector, which was used to distinguish FS surface EMG signals from FP surface EMG signals.

The following paragraphs were firstly to explain the scheme of acquiring surface EMG signal; then to introduce the method of calculating WCE feature vector and recognizing FS and FP pattern; lastly to analyze and discuss the research results. 


\section{SURFACE EMG SIGNAL'S ACQUISITION}

All surface EMG signals were recorded by Metr-1-10UK (made by Mega Electronics Ltd) in the EMG room at Hua Shan Hospital in Shanghai, China. Because the power density function of SEMG signal outside the range from $5-10 \mathrm{~Hz}$ to $400-450 \mathrm{~Hz}$ has negligible contributions [10], the low cut-off frequency and the high cut-off frequency of Metr-1-10UK were set $5 \mathrm{~Hz}$ and $500 \mathrm{~Hz}$ respectively. The sampling frequency $f_{s}$ was determined at $1000 \mathrm{~Hz}$. It was about $2 \mathrm{~cm}$ between two measuring surface electrodes $($ diameter $=5 \mathrm{~mm})$ which were put on the skin surface over the pronator teres in the right forearm along the flexor. And the ground electrode (denoted the capital letter "G") was on the flexor carpi radialis and the palmaris longus (see Figure 1 for their arrangement). The negative electrode (denoted by the symbol "-") was placed nearer subject's heart than the positive electrode (denoted by the symbol "+") to form a differential comparator amplifier.

During every acquiring process, every subject was instructed to do two different kinds of limb actions: forearm supination (FS) and forearm pronation (FP). The whole acquiring process was divided into three stages: preparing stage, acting stage and sustaining stage. At the preparing stage, every subject put his right forearm on the measure platform flatly and naturally (see Figure 1 (b)). At the acting stage, there were two cases: FP and FS. In the process of FP, the forearm was quickly transformed from the pose at Figure 1(b) to that at (a), and in the process of FS, the forearm was quickly transformed from the pose Figure 1(b) to that at (c). The whole process of FS or FP must be finished within 0.5 second. After finishing FS or FP, every subject kept the end condition of the limb actions for one or two seconds, the stage was the sustaining stage.

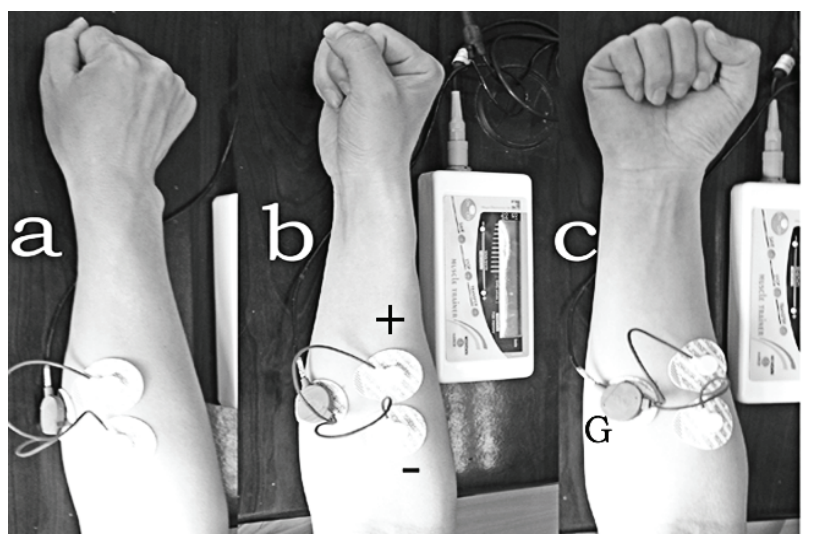

Figure 1. The forearm posture. (b): the forearm posture before forearm actions; (a) and (c) are respectively the posture after forearm pronation and forearm supination; +, -, G represent respectively the positive, negative electrodes and the ground.

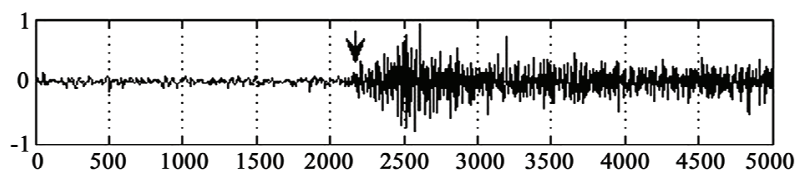

(a) Time (ms)

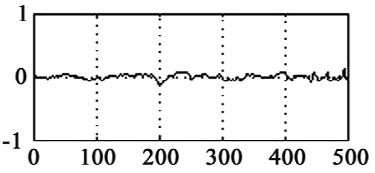

(b) Time (ms)

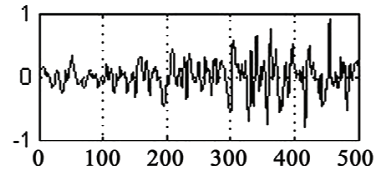

(c) Time (ms)
Figure 2. Raw surface EMG signal and its segments (a): Raw surface EMG signal; (b) and (c) are respectively the segments of the raw surface EMG signal on (a) at preparing stage and acting stage. The arrow on (a) points the start of the acting stage.

30 healthy subjects participated in study. Two sets of surface EMG signals (FS and FP) were respectively recorded from every subject's forearm flexor. Figure 2 showed the wave of a set of recorded surface EMG signal. The arrow at Figure 2 pointed to the start time of forearm action. The start time could be determined by an amplitude criterion [11]. A 50-points window slid along the surface EMG signal from left to right. At the same time, the number of the points above one threshold in the window was calculated. The first time when the number was above 10 was regarded as the starting time of forearm action.

After the start time of forearm actions and immediate to the start time, one 0.5 -second surface EMG signal (500 samples) was segmented from every raw surface EMG signal, see Figure 2(c).Thus, 60 segments of surface EMG signals obtained in all, there were two surface EMG signal patterns: FS and FP, 30 sets for each pattern.

\section{WAVELET COEFFICIENT ENTROPY}

Multiresolution analysis was first proposed in 1989 by Mallat [12]. Since then, the advanced research and development in wavelet analysis have applied in many fields such as signal processing and pattern recognition [13]. Wavelet packets were introduced by Coifman and Wickerhauser (1992) [14] as a generalized family of multiresolution orthogonal or biorthognal basis. Unlike wavelet transform which is realized only by a low-pass filter bank, wavelet packet transform is implemented by a basic two-channel filter bank which can be iterated over either a low-pass or a high-pass branch. So the information in high frequencies can be analyzed as well as that in low frequencies in wavelet packet transform. As a result, finer frequency bands can be gained by wavelet packet transform than by wavelet transform. Therefore, WPT has been widely applied in biomedical signal analysis and many encouraging results have been obtained $[15,16]$.

Given a finite energy signal, $\mathrm{s}(\mathrm{t})$, whose scaling space 
is assumed as $U_{0}^{0}$, wavelet packet transform can decompose $U_{0}^{0}$ into small subspaces $U_{-j}^{n}$ in dichotomous way.

The dichotomous way is realized by the following recursive scheme.

$$
U_{-j-1}^{n}=U_{-j}^{2 n} \oplus U_{-j}^{2 n+1}, j \in Z ; n \in Z_{+}
$$

where $j$ is the resolution level and $\oplus$ denotes orthogonal decomposition. $U_{-j-1}^{n}, U_{-j}^{2 n}$ and $U_{-j}^{2 n+1}$ are three close spaces corresponding to $u_{n}(t), u_{2 n}(t)$ and $u_{2 n+1}(t)$. $u_{n}(t)$ satisfies the following equation [14]

$$
\left\{\begin{array}{c}
u_{2 n}(t)=\sqrt{2} \sum_{k \in Z} h(k) u_{n}(2 t-k) \\
u_{2 n+1}(t)=\sqrt{2} \sum_{k \in Z} g(k) u_{n}(2 t-k)
\end{array}\right.
$$

where the function $u_{0}(t)$ can be identified with the scaling function $\varphi$ and $u_{l}(t)$ with the mother wavelet $\psi . h(k)$ and $g(k)$ are the coefficients of the low-pass and the high-pass filters respectively. The sequence of function $\left\{u_{n}\right\} \quad(n=0,1, \ldots \infty)$, which is generated from a given function $u_{0}$ by (2), is called wavelet packet basis function. Figure 3 shows the WPT tree.

When a finite energy signal, $s(t)$, is decomposed to the fourth resolution level $(j=4)$ with wavelet packet transform, the whole scaling space $U_{0}^{0}$ with frequencies in the interval $\left(0,2^{-1} f_{s}\right)$ is divided into 16 subspaces with frequencies correspondingly in the interval $\left((n-1) 2^{-j-1} f_{s}\right.$, $n 2^{-j-1} f_{s}, n=1,2, \ldots, 16$. The sub-signal at $U_{-j}^{n-1}$, the $n$th subspace on the $j$ th level, can be reconstructed by

$$
s_{j}^{n}(t)=\sum_{k} D_{k}^{j, n} \psi_{j, k}(t), k \in Z
$$

where $\psi_{j, k}(t)$ is the wavelet function, $D_{k}^{j, n}$ was the wavelet packet coefficients at $U_{j}^{n-1}$ and it is calculated by the recursive formula

$$
\left\{\begin{array}{l}
D_{k}^{j, 2 n}=\sum_{l \in Z} D_{l}^{j-1, n} h(l-2 k) \\
D_{k}^{j, 2 n+1}=\sum_{l \in Z} D_{l}^{j-1, n} g(l-2 k)
\end{array}\right.
$$

where, $\mathrm{k}$ is the $k$ th wavelet packet coefficient at each subspace on the $j$ th level, $\mathrm{k}=1,2, \ldots, \mathrm{K}, \mathrm{K}=500$ (samples) $12^{\mathrm{j}}$.
Thus, the finite energy signal, $s(t)$ can be reconstructed as

$$
s(t)=\sum_{n=1}^{2^{-j}} s_{j}^{n}(t)=\sum_{n=1}^{2^{-j}} \sum_{k} D_{k}^{j, n} \psi_{j, k}(t)
$$

After surface EMG signal $\mathrm{s}(\mathrm{t})$ was decomposed into 16 FB by wavelet packet transform. The wavelet packet coefficient in the $n$th $\mathrm{FB}$ was assumed as

$$
D_{n}=\left\{d_{n}(k), k=1,2, \cdots, K\right\}
$$

Here, $k$ symbolizes time too. And then, these coefficient functions were assembled and normalized to a coefficient matrix.

$$
D=\left\{\begin{array}{l}
D_{1} \\
D_{2} \\
\ldots \\
D_{2^{j}}
\end{array}\right\}=\left\{\begin{array}{l}
d_{1}(1), d_{1}(2), \ldots, d_{1}(K) \\
d_{2}(1), d_{2}(2), \ldots, d_{2}(K) \\
\ldots \\
d_{2^{j}}(1), d_{2^{j}}(2), \ldots, d_{2^{j}}(K)
\end{array}\right\} \mathrm{j}=4
$$

After normalized, the values in coefficient matrix were within $[-1,1]$. The interval $[-1,1]$ was decomposed into $M$ regions with identical size

$$
\left[-1, a_{1}\right),\left[a_{1}, a_{2}\right), \ldots, \quad\left[a_{m-1}, a_{m}\right), \ldots,\left[a_{M-1}, 1\right] .
$$

Supposed the number of $\mathrm{d}_{\mathrm{n}}(\mathrm{k})$ within $\left[a_{m-1}, a_{m}\right)$ was $N$, thus, a probability of the $m$ th region could be calculated

$$
p_{n}(m)=N / K \text {. }
$$

WCE of the $n$th FB was

$$
W C E(n)=-\sum_{m=1}^{M} p_{n}(m) \ln \left[p_{n}(m)\right]
$$

Englehart K. (2003) [17] combined WPT and principal components analysis to get a WPT features. The WPT features could get much lower error identification rate than the features gained by conventional methods. So the WPT features were adopted to compare with

\begin{tabular}{|c|c|c|c|c|c|c|c|c|c|}
\hline \multicolumn{10}{|c|}{$U_{0}^{0}$} \\
\hline \multicolumn{6}{|c|}{$U_{-1}^{0}$} & \multicolumn{4}{|c|}{$U_{-1}^{1}$} \\
\hline \multicolumn{4}{|c|}{$U_{-2}^{0}$} & \multicolumn{2}{|c|}{$U_{-2}^{1}$} & \multicolumn{2}{|c|}{$U_{-2}^{2}$} & \multicolumn{2}{|c|}{$U_{-2}^{3}$} \\
\hline & $\begin{array}{c}0 \\
-3\end{array}$ & & & $U_{-3}^{2}$ & $U_{-3}^{3}$ & $U_{-3}^{4}$ & $U_{-3}^{5}$ & $U_{-3}^{6}$ & $U_{-3}^{7}$ \\
\hline$U_{-4}^{0}$ & $U_{-4}^{1}$ & $U_{-4}^{2}$ & $U_{-4}^{3}$ & $U_{-4}^{4} \quad U_{-4}^{5}$ & $U_{-4}^{6} \quad U_{-4}^{7}$ & $U_{-4}^{8} \quad U_{-4}^{9}$ & $U_{-4}^{10} \quad U_{-4}^{11}$ & $U_{-4}^{12} \quad U_{-4}^{13}$ & $U_{-4}^{14} \quad U_{-4}^{15}$ \\
\hline
\end{tabular}
WCE features in this paper.

The WCE features and the WPT features were respectively used to identify FP surface EMG signal and FS surface EMG signal, and the error identification rate with the increase of signal's sampling points was computed.

In order to effectively remove noise from SEMG signal, we should make the MUAPs from one muscle in charge of one kind of limb actions centralize on one

Figure 3. The tree structure of wavelet packet transform ( $U_{-j}^{n-1}$ shows the $n$th subspace the $j$ th resolution level). 
narrow frequency band as much as possible, rather than make them spread out over one wide frequency band. It is well known that the information carried by the coefficients of WPT depends on the joint characteristics of the analyzed signal and the selected wavelet function; the more similar are the two functions, the less spread are the significant coefficients in the time scale plane. Because Daubechies family of wavelet packets most seems to resemble MUAPs [18] and the simplest of these wavelets is $\mathrm{db} 2, \mathrm{db} 2$ is adopted as the mother wavelet. At the same time, Martha Flanders (2002) [18] pointed out that the length of the $\mathrm{db} 2$ at the forth level resolution was approximately the length of a MUAP.

\section{THE ERROR DECISION RATE BASED ON BAYES DECISION}

Let $\omega_{1}$ and $\omega_{2}$ be the two classes (FS and FP ASEMG signal patterns) to which our patterns belong. Feature vector $x$ represents an unknown pattern. The Bayes rule is

$$
P\left(\omega_{i} / x\right)=\frac{p\left(x / \omega_{i}\right) P\left(\omega_{i}\right)}{\sum_{i=1}^{2} p\left(x / \omega_{i}\right) P\left(\omega_{i}\right)}
$$

$p\left(\omega_{1} / x\right)$ is the $i$ th conditional probability. $p\left(\omega_{1} / x\right)$ is the class-conditional probability density function, see the two curves at Figure 3. $p\left(\omega_{i}\right)$ is priori probability. In this paper, $p\left(\omega_{1}\right)=p\left(\omega_{2}\right)=1 / 2$.

The Bayes classification rule can be stated as

If $p\left(\omega_{1} / x\right)>p\left(\omega_{2} / x\right), x$ is classified to $\omega_{1}$

If $p\left(\omega_{1} / x\right)<p\left(\omega_{2} / x\right), x$ is classified to $\omega_{2}$

If the straight line at $\mathrm{x}_{0}$ is the threshold partitioning the feature space into regions: $\mathrm{R}_{1}$ and $\mathrm{R}_{2}$ (see Figure 3), all values of $x$ in $\mathrm{R}_{1}$ are classified as $\mathrm{w}_{1}$, and all values of $x$ in $\mathrm{R}_{2}$ are classified as $\mathrm{w}_{2}$. It is obvious that decision errors are unavoidable. The total probability, Pe, of committing a decision error is given by

$$
P_{e}=\frac{1}{2} \int_{-\infty}^{x_{o}} p\left(x / \omega_{2}\right) d x+\frac{1}{2} \int_{x_{0}}^{\infty} p\left(x / \omega_{1}\right) d x
$$

which is equal to the total shaded area under the curves in Figure 4. So the error decision rate is calculated by

$$
R_{e}=P_{e} \times 100 \%
$$

\section{RESULT}

Some WCE(n) were chosen to form WCE feature vector, which was used to distinguish FS surface EMG signals from FP surface EMG signals. It was found that the WCE feather vector consisted of $\mathrm{WCE}(7)(187.25 \mathrm{~Hz}$, $218.75 \mathrm{~Hz})$ and $\mathrm{WCE}(8)(218.75 \mathrm{~Hz}, 250 \mathrm{~Hz})$ can more effectively recognize $\mathrm{FS}$ and FP patterns than other

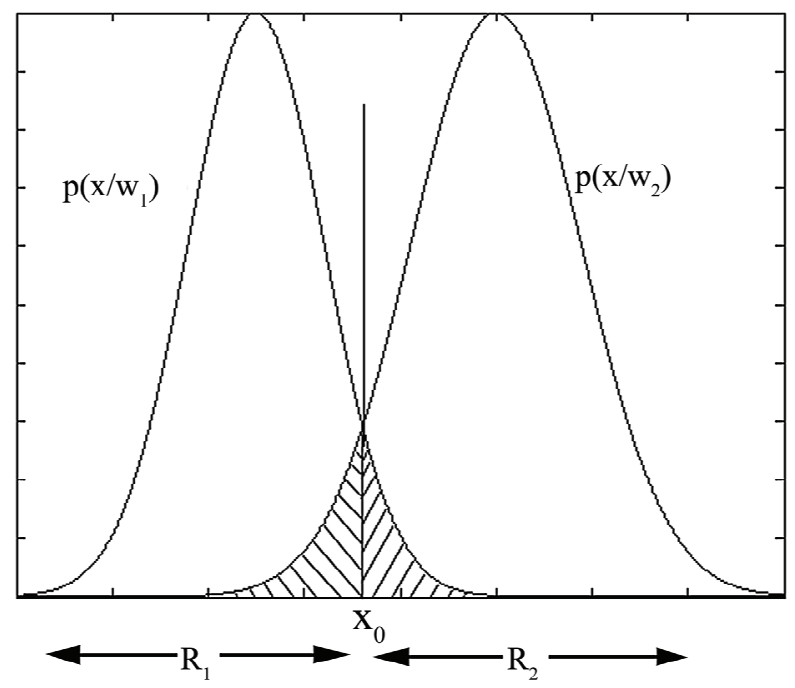

Figure 4. Example of the two regions $\mathrm{R}_{1}$ and $\mathrm{R}_{2}$ formed by the Bayesian classifier for the case of two classes. The straight line at $\mathrm{x}_{0}$ is a threshold of $\mathrm{R}_{1}$ and $\mathrm{R}_{2} . \mathrm{p}\left(\mathrm{x} / \mathrm{w}_{1}\right)$ and $\mathrm{p}\left(\mathrm{x} / \mathrm{w}_{2}\right)$ are respectively the class-conditional probability density function at regions: $R_{1}$ and $R_{2}$.

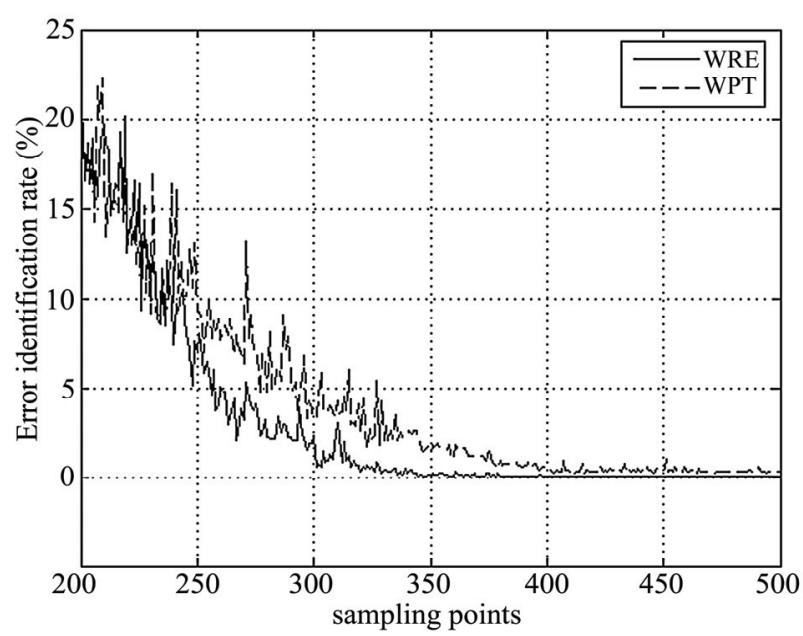

Figure 5. The error decision rate based on bayes decision VS the sampling points of initial signal.

WCE feature vector. Therefore, $\operatorname{WCE}(7)$ and $\operatorname{WCE}(8)$ were chosen to constitute a 2 dimensionality WCE feature. Based on Bayes decision, both WCE feature and WPT feature were employed to recognize FP and FS surface EMG signal. Figure 5 depicted the error identification rate vs. initial signal sampling points. The curves on Figure 5 were the real error decision rate to the sampling points. With the increasing of the sampling points, the signal undoubtedly included more and more feature information, so the error decision rate decreased with the increasing of the sampling points. However, from Figure 5, we found another result that the WCE feature performed better than the WPT feature. When the sampling points were between 200 and 500, the error decision rate by the WCE features was lower than by the 
WPT features. Furthermore, when the sampling points was above 350 , the error decision rate by the WCE features was almost 0 .

\section{DISCUSSION}

In pattern recognition [19], a feature vector was insisted of some features. The number of the features in the feature vector was called as dimensionality. In general cases, whether one pattern of signal could be effectively and accurately identified depended much upon two important factors. One was a set of optimal features. A set of desired features not only contain the characteristic information which characterize one pattern of signals, but also ignore the particular information, which only existed in some individual signals or sometime might be the result of noisy measurements, and the general information, which exist in all pattern signals. From the results in this paper, both WCE feature and WPT feature could capture the characteristic information of one pattern of surface EMG signal.

In this paper, wavelet packet coefficients matric D (see Eq.7) can be rewrote as a 16X63 matric

$$
D=\left[\begin{array}{l}
d_{1}(1), d_{1}(2), \ldots, d_{1}(63) \\
d_{2}(1), d_{2}(2), \ldots, d_{2}(63) \\
\ldots \\
d_{16}(1), d_{16}(2), \ldots, d_{16}(63)
\end{array}\right]
$$

Find some orthonormal matrix $\mathrm{P}$ in $\mathrm{Y}=\mathrm{PD}$ such that $\mathrm{C}_{\mathrm{Y}}$, the covariance matrix of $\mathrm{Y}$, is a diagonal matrix.

$$
C_{Y}=P C_{D} P^{T}=\left[\begin{array}{ccccc}
\lambda_{1} & 0 & 0 & \ldots & 0 \\
0 & \lambda_{2} & 0 & \ldots & 0 \\
0 & 0 & \lambda_{3} & \ldots & 0 \\
0 & 0 & 0 & \ldots & \lambda_{63}
\end{array}\right]
$$

Here, the rows of $\mathrm{P}$ are the principal components of $\mathrm{D}$. $C_{D}$ is the covariance matrix of D. $\lambda_{1}>\lambda_{2}>\lambda_{3}>\ldots$

According to the following criterion, eight bigger $\lambda_{i}$ form WPT feature vector.

$$
T H=\frac{\sum_{i=1}^{M} \lambda_{i}}{\sum_{i=1}^{63} \lambda_{i}}>\text { Threshold }
$$

When $M=8, T H>0.934$. If $M>=9, T H$ increase very weeny.

Obviously, WPT feature vector included the information about the whole frequency band $(0 \mathrm{~Hz}-500 \mathrm{~Hz})$.

At the other hand, the WCE features were got from only two narrower FBs, so the WCE features were rationally considered including less common information of surface EMG signal and noise than WPT features. The other was dimensionality reduction. There was more than one reason for the necessity to reduce the number of features to a sufficient minimum [19]. Computational complexity was the obvious and important one. The computational complexity for pattern recognition was reduced as dealing with the features in a lower dimensional space. In this paper, WCE feature vector only needed two features, but in WPT feature vector, we used 8 features.

\section{CONCLUSIONS}

WCE was a more effective method to extract feature from surface EMG signal than the combination of WPT and principal components analysis. FS surface EMG signals could be successfully and rapidly distinguished from FP surface EMG signals by the WCE features.

\section{ACKNOWLEDGEMENTS}

The research was funded by the educational science program of Guangzhou during the 11th Five-Year Plan Period (No.07B171and 07B258) and Guangdong Natural Science Fund (No. 7301261).

\section{REFERENCES}

[1] Stokes, I.A.F. (2005) Relationships of EMG to effort in the trunk under isometric conditions force-increasing and decreasing effects and temporal delays. Clinical Biomechanics, 20, 9-15.

[2] Wakeling, J.M. (2009) Patterns of motor recruitment can be determined using surface EMG. Journal of Electromyography and Kinesiology, 19, 199-207.

[3] Kaplanis, P.A., Pattichis, C.S., Hadjileontiadis, L.J., Roberts, V.C. (2009) Surface EMG analysis on normal subjects based on isometric voluntary contraction. Journal of Electromyography and Kinesiology, 19, 157-171.

[4] Lei, M., Wang, Z.Z., Feng, Z.J. (2001) Detecting nonlinearity of action surface EMG signal. Physics Letters A, 290, 297-303.

[5] Englehart, K., Hudgins, B., Parker, P.A., Stevenson, M. (1999) Classification of the myoelectric signal using time-frequency based representations. Medical Engineering \& Physics, 21, 431-438.

[6] Hudgins, B., Parker, P., Scott, R.N. (1993) A new strategy for multifunction myoelectric control. IEEE Trans. Biomed. Eng., 40(1), 82-94.

[7] Stashuk, D. (2001) EMG signal decomposition: how can it be accomplished and used? Journal of Electromyography and Kinesiolugy, 11, 151-173.

[8] Hu, X. and Wang, Z.Z. (2004) Detecting the motor unit action potential from surface EMG signals based on wavelet transform, 2004 IEEE International workshop on Biomedical circuits \& system, S2. 6-15.

[9] Farina, D. and Merletti, R. (2001) A novel approach for presice simulation of the EMG signal detected by surface electrodes. IEEE Trans. Biomed. Eng., 48(6), 637-646.

[10] Merletti, R. and Torino, P.D. (1999) Standards for Re- 
porting EMG Data. Journal of Electromyography and Kinesiolugy.

[11] Hu, X., Yu, P., Yu Q., Liu, W.X., Qin, J. (2008) Classification of Surface EMG Signal Based on Energy Spectra Change, 2008 International Conference on BioMedical Engineering and Informatics, 375-379.

[12] Mallat, S.G. (1989) A theory for multiresolution signal decomposition: the wavelet representation. IEEE Trans. Pattern Anal. Mach. Intell, 11, 674-693.

[13] Daubechies, I., Mallat, S., Willsky, A.S. (1992) Introduction to the special issue on wavelet transforms and multiresolution signal analysis. IEEE Trans. Inform. Theory, 38, 529-531.

[14] Coifman, R.R. and Wickerhauser, M.V. (1992) Entropybased algorithms for best basis selection. IEEE Trans.
Inform. Theom, 38, 713-718.

[15] Karlsson, S., Yu, J., Akay, M. (1999) Enhancement of spectral analysis of myoelectric signals during static contractions using wavelet methods. IEEE Trans. Biomed. Eng., 46, 670-684.

[16] Flanders, M. (2002) Choosing a wavelet for single-trial EMG. Journal of Neuroscience Methods, 116, 165 -177.

[17] Englehart, K. and Hudgins, B. (2003) A robust, real-time control scheme for multi-function myoelectric control IEEE Trans. Biomed. Eng., 50(7), 848-854.

[18] Flanders, M. (2002) Choosing a wavelet for single-trial EMG. Journal of Neuroscience Methods, 116, 165-177.

[19] Sameer, S., Nabeel, M., Walter, K. (2001) Advances in pattern recognition. Springer. 